\section{The behavior of rats during copulation as a function of prior copulatory experience*}

\author{
DIRK W. MOSIG and DONALD A. DEWSBURY $\dagger$ \\ University of Florida, Gainesville, Florida 32601
}

The behavioral patterns accompanying the copulatory behavior of nine male rats with no prior adult copulatory experience were compared with those of nine males that had experienced four prior tests. Age was controlled. Effects of experience on standard measures of copulatory behavior were similar to those in earlier work. There were no significant differences in other behavioral patterns as a function of experience. Thus, the tendency to display various behavioral patterns during copulatory activity appears completely formed prior to the first such activity and generally unaffected by adult copulatory experience.

As the copulatory pattern of laboratory rats has been the subject of intensive experimental analysis, there has arisen much interest in the effects of various prior experiences on the complete adult pattern. It has been shown that under some conditions early social isolation can interfere with adult copulatory behavior (Folman \& Drori, 1965; Gerall, Ward, \& Gerall, 1967; Gruendel \& Arnold, 1969). Postcastration copulatory behavior in rats appears unaffected by prior adult copulatory experience (Stone, 1927; Rabedeau \& Whalen, 1959). Various aspects of the normal copulatory patterns of cats (Rosenblatt, 1965), mice (McGill, 1962), and hamsters (Bunnell \& Kimmel, 1965) have been shown to be altered by copulatory experience.

Effects of prior copulatory experience on the normal copulatory pattern of laboratory rats has been studied by Larsson (1959) and Dewsbury (1969). In general, the rate of copulation is increased by experience, as indicated particularly by decreased mount, intromission, and ejaculation latencies and increased intromission rates. The effects are not large relative to individual differences and are not always statistically significant, but they are remarkably consistent. For example, the data on intromission rates (intromissions/minute) revealed that experienced rats intromitted at a faster rate than inexperienced rats in all 19 comparison. Yet only seven of these comparisons were statistically significant; none of the four comparisons in the first series were significant.

Dewsbury (1967) has pointed out that

* This research was supported by Grant GB-12941 from the National Science Foundation. Hormones were provided through the courtesy of Dr. Preston L. Perlman of the Schering Corporation, Bloomfield, New Jersey.

$\dagger$ Please address requests for reprints to: D. A. Dewsbury, Department of Psychology, 109 Building E, University of Florida, Gainesville, Florida 32601. intromissions occupy approximately $1 \%$ of a typical test of copulatory behavior in rats. A system for quantifying the remaining $99 \%$ of the behavior was proposed. All the data in that study were from experienced animals. The effects of copulatory experience on these behavioral patterns have not been studied. The purpose of the present research was to combine the approaches of Dewsbury (1967) and Dewsbury (1969) to assess the effects of copulatory experience on the behavior accompanying copulation.

\section{METHOD}

The Ss were 23 male Long-Evans rats purchased from a commercial supplier and approximately 90 days of age at the beginning of the experiment. They had been raised in all-male groups from weaning and were without prior copulatory experience. They were mated with a total of 48 females of the same age and strain.

Tests were conducted in circular Plexiglas arenas approximately $81 \mathrm{~cm}$ in diam and $66 \mathrm{~cm}$ high. The arenas were placed on wooden bases covered with a commercially produced litter material. Behavioral events were recorded manually on an operations recorder.

The animals were assigned at random to two groups with Ns of 11 and 12, which were designated Groups A and B. Two Ss from Group A and three Ss from Group B failed to copulate in four successive tests, thus reducing the effective $\mathrm{N}$ in each group to nine. All animals received two sessions of handling and exploration of the test arenas before the beginning of testing. Group A then received five positive tests of copulatory behavior spaced at least 1 week apart. Group B received just one test, at the same time as the animals of Group A received their fifth test. Thus, it was possible to compare the behavior of an experienced group (A) with that of an inexperienced group (B) at the same age and under identical testing conditions.

On each test the males were given $5 \mathrm{~min}$ to adapt to the test arena before a receptive female was introduced to begin the test. Tests were then terminated at the end of $15 \mathrm{~min}$ if no intromissions occurred or at the occurrence of the second ejaculation if copulation was initiated.

Females were prepared with injections of $0.1 \mathrm{mg}$ estradiol benzoate $75 \mathrm{~h}$ before testing and $1.0 \mathrm{mg}$ progesterone $3-6 \mathrm{~h}$ before testing. Only receptive females, as assessed on each test day with nonexperimental males, were used in tests.

\section{MEASURES}

' Copulatory behavior in rats occurs in bouts or "series" of mounts without vaginal penetration, mounts with intromission (intromissions), and mounts with intromission and ejaculation (ejaculations). Each complete series is terminated by an ejaculation. The standard measures of copulatory behavior patterned after Beach \& Jordan (1956) were used. These included: mount latency (ML)-latency from introduction of the female to the first mount with thrusting; intromission latency (IL)-latency from introduction of the female to the first intromission; ejaculation latency (EL)-latency from first intromission to an ejaculation; intromission frequency (IF)-number of intromissions in a series; mount frequency(MF)-number of mounts in a series; intromission rate (IR)-number of intromissions per minute within a series; and postejaculatory interval (PEI)-latency from ejaculation to the first intromission of the subsequent series.

The duration of each of seven other mutually exclusive categories of behavior were recorded. These included: (1) pursuit mount-the male follows the female, remaining oriented toward her, including mounting but excluding behavior fitting other categories; (2) locomotorexploratory behavior-the male moves about the cage, rearing, manipulating feces or sawdust, or appearing to explore anything except the female; (3) genital grooming-the male grooms his genital region in a stereotyped posture generally resting on haunches and bending the head straight down; (4) nongenital grooming-all grooming and scratching not included under genital grooming; (5) sniffing female-the male approaches the female and appears to sniff part of her body while remaining oriented with his head toward her body but with no mounting movements; (6) standing still-the male remains motionless. This category is somewhat broader than the "immobility" category of Dewsbury (1967); (7) lying down-self explanatory.

In summarizing data from these categories, the general method of Dewsbury (1967) was followed. The EL was divided into fifths and the PEI into 
Table 1

Mean Scores on Standard Measures of Copulatory Behavior and Results of $t$ Tests

\begin{tabular}{lcccc}
\hline & \multicolumn{2}{c}{ Mean Score } & & \\
\cline { 2 - 3 } Measure $^{1}$ & Group A & Group B & $\mathrm{t}$ & $\mathrm{p}$ \\
\hline IL & 2.94 & 126.9 & 1.10 & n.s. \\
IL & 19.4 & 126.9 & 1.10 & n.s. \\
EL & 547.2 & 727.3 & 1.15 & n.s. \\
IF & 9.9 & 9.0 & 0.72 & n.s. \\
MF & 6.1 & 6.3 & 0.08 & n.s. \\
IR & 2.17 & 1.67 & 0.93 & n.s. \\
PEI & 391.2 & 415.1 & 0.58 & n.s. \\
\hline
\end{tabular}

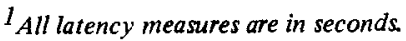

quarters. The percent of time spent by each animal in each of the 10 periods (IL, $5 \mathrm{EL}$ periods and $4 \mathrm{PEI}$ periods) was determined. As there were few instances of lying down, these data were combined with those of standing still.

\section{RESULTS}

Data on standard measures of copulatory behavior of the experienced (Group A) and inexperienced (Group B) rats appear in Table 1 . The experienced animals showed lower mean scores on all latency measures and higher IRs, although none of the comparisons was statistically significant.

The means and standard errors of the categorization data appear in Fig. 1. Significance of differences was assessed with $t$ tests for the entire IL, EL, and PEI periods. There were no significant differences between experienced and inexperienced rats.

\section{DISCUSSION}

The data on the standard measures of ML, IL, EL, and IR are in good agreement with those of Larsson (1959) and Dewsbury (1969). There now are five similar tests and in all five instances $\mathrm{ML}$, $I L$, and EL and IR in the first series are in the same direction. That is a total of 20 comparisons. Of the 10 comparisons of $E L$ and IR in the first series, all are in the same direction; none show significant differences. The reason for this probably lies in reliable individual differences which must of necessity be treated as error variance in any study of the effects of experience in which age is controlled (cf. Dewsbury, 1969).

Somewhat more remarkable was the fact that the behavioral patterns accompanying

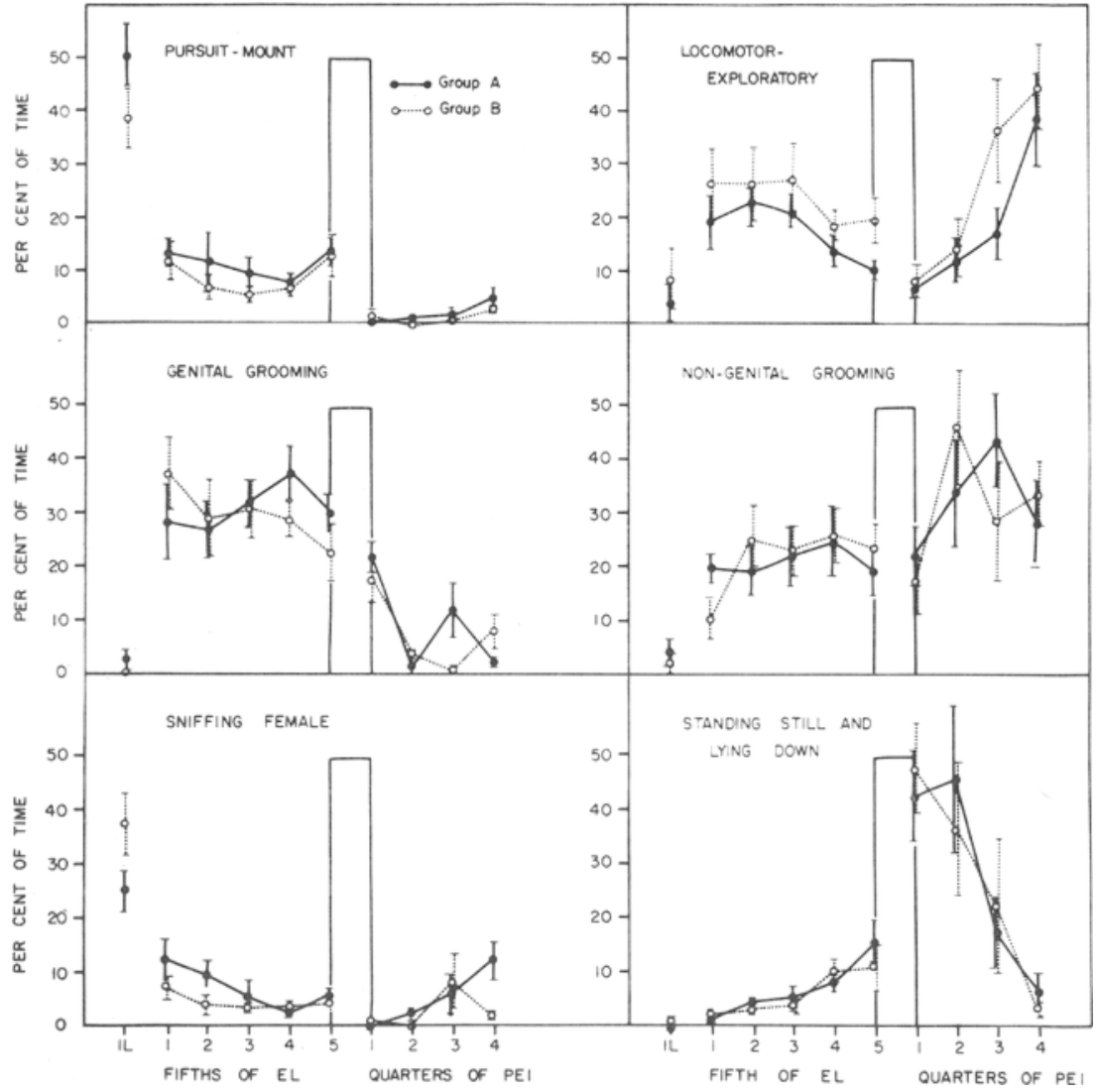

Fig. 1. Mean percent of time spent in each of six behavioral patterns during the intromission latency period, successive fifths of the ejaculation latency period, and successive quarters of the postejaculatory interval as a function of experience. Group $A$ had four prior tests; Group B had none. One standard error is shown for each measure. copulation in experienced and inexperienced males were indiscriminable. It had been anticipated that this more detailed analysis of behavior would reveal differences not detected with the standard measures of copulatory behavior. It was expected, for example, that inexperienced animals might begin pursuing the females earlier in the PEI. This was not the case in the present data. As experienced males had spent much more time in the arenas, it was anticipated that their locomotorexploratory behavior might be reduced relative to that of inexperienced males. Although this difference appeared slightly more appreciable than others, it still did not approach statistical significance.

It, thus, would appear that with a detailed analysis of the total behavioral repertoire of the male rat, no effects of adult copulatory experience are demonstrable in the present situation. The dispositions toward such behavior must be fully developed as a result of the genotype of the animal and its interaction with environmental factors in development prior to the first copulatory test. To the extent that such development is dependent upon contact with females, this must take place prior to weaning, as these males had no contact with females between weaning and testing. Precopulatory experience (preweaning hetero- and homosexual and postweaning homosexual) may affect the total behavioral pattern displayed during copulation, and it is here that experimental analysis may reveal appreciable effects.

\section{REFERENCES}

BEACH, F. A., \& JORDAN, L. Sexual exhaustion and recovery in the male rat. Quarterly Journal of Experimental Psychology, 1956, 8, 121-133.

BUNNELL, B. N., \& KIMMEL, M. E. Some effects of copulatory experience on postcastration mating behavior in the male hamster. Psychonomic Science, 1965, 3 179-180.

DEWSBURY, D. A. A quantitative description of the behavior of rats during copulation. Behaviour, 1967, 29, 154-178.

DEWSBURY, D. A. Copulatory behavior of rats (Rattus norvegicus) as a function of prior copulatory experience. Animal Behaviour, $1969,17,217.223$

FOLMAN, Y., \& DRORI, D. Normal and aberrant copulatory behavior in male rats $(R$. norvegicus) reared in isolation. Animal Behaviour, 1965, 13, 427-429.

GERALL, H. D., WARD, I. L., \& GERALL, A. A. Disruption of the male rat's sexual behaviour induced by social isolation. Animal Behaviour, 1967, 15, 54-58.

GRUENDEL, A. D., \& ARNOLD, W. J. Effects of early social deprivation on reproductive behavior of male rats. Journal of Comparative \& Physiological Psychology, 1969, 67, 123-128.

LARSSON, K. Experience and maturation in the development of sexual behavior in the male puberty rat. Behaviour, 1959, 14, 101-107.

McGILL, T. E. Reduction in "headmounts" in 
the sexual behavior of the mouse as a function of experience. Psychological Reports. 1962. $10,284$.

RABEDEAL, R. G., \& WHALEN, R, F, lffects of copulatory experience on mating behavior of the male rat. Journal of Comparative \& Physiological Psychology, 1959, 52, 482-484.
ROSI NBLATT, J. S. Hifects of experience on exual behavior in male cats. In 1. A. Beach (Ld.) Sex and behavior. New York: Wiley, 1965. Pp. 413-439.

STONF, C.P. The retention of copulatory ability in male rats following castration. Joumal of Comparative Psychology, 1927, 7, 269-287.

\title{
Injections of neurohumors in the cat thalamus and visual discrimination performance
}

\author{
DAVID D. AVERY, Colorado State University, Fort Collins, Colo. 80521 \\ and \\ DWIGHT M. NANCE, University of Houston, Houston, Tex. 77004*
}

\begin{abstract}
The influences of central applications of adrenergic and cholinergic stimulating and blocking agents to the cat thalamus on successive visual discrimination performance were investigated. Applications of both noradrenaline and the adrenergic blocking agent ethobutamoxane improved discrimination performance by reducing nonreinforced responding. Conversely, injections of both the cholinergic stimulator and blocker disrupted visual discrimination. Carbachol reduced responses to the discriminative stimulus without affecting nonreinforced response rate, and atropine sulfate increased nonreinforced responses without changing the rate of reinforced responding.
\end{abstract}

The center median nucleus of the thalamus is one of the nonspecific thalamic nuclei that provide a thalamocortical extension of the brainstem reticular system in the cat. The thalamic-brainstem continuum appears to supply certain integrative or motivational functions that are essential for acquisition, retention, and performance of new behavioral patterns. Support for this notion derives from studies showing changes in performance measures as a result of altering reticular function by electrical stimulation, ablation, or, more importantly for the present investigation, chemical stimulation (Zuckerman, 1959; Warren \& Ackert, 1960; Grossman et al, 1965). Grossman and his colleagues $(1965,1966,1968)$, in a series of experiments, have demonstrated that cholinergic stimulation or blockage of both thalamic midline and reticular nuclei in the rat can greatly affect acquisition and asymptotic performance in both appetitive and aversive test situations. In addition, Avery \& Nance (1970) found that injections of adrenergic, as well as cholinergic, substances into the center median nucleus of the cat thalamus can selectively inhibit or facilitate simple appetitive operant responding. Adrenergic stimulation and cholinergic blockage increased response rate, whereas cholinergic stimulation and adrenergic blockage had an opposite inhibitory influence.

*Presently a postdoctoral fellow at the Worcester Foundation for Experimental Biology, 222 Maple Avenue, Shrewsbury, Mass. 01545.
Since ascending cholinergic and aminergic pathways through the brainstem have been identified in the cat, as well as the rat, and in view of the previous findings in both species with thalamic injections of neurohumors, the investigation to be reported was an attempt to unravel the functional significance of these chemical circuits in the performance of newly acquired, complex behavioral patterns. More specifically, the experiments were concerned with the effects of direct injections of adrenergic and cholinergic stimulating and blocking agents in the cat thalamus on complex visual discrimination.

\section{METHOD}

A double-wall cannula was directed at the center median nucleus of the thalamus in each of 13 mature mongrel cats, weighing between 6 and 9 lbs. The implant was secured to the skull and anchor screws with dental cement, following standard procedures. Each animal was housed individually in a constantly illuminated, tem perature-controlled room $\left(22.0^{\circ} \pm 1.0^{\circ} \mathrm{C}\right)$. Injections were made with the aid of a micromanipulator and a Hamilton microsyringe, which was attached to the internal cannula via polyethylene tubing. A dummy cannula, constructed from stainless steel wire, was in place except during injections. The injection cannula was of the same length and diameter as the dummy cannula.

The substances applied intracranially and their respective dosages were the same as in a previous study (Avery \& Nance,
1970) and consisted of l-arterenol bitartrate (noradrenaline) ( $40 \mathrm{micrograms} / \mathrm{microliter}$ ), ethobutamoxane (13.5 micrograms/ microliter), carbamylcholine chloride (carbachol) (8 micrograms/microliter), and atropine sulfate (50 micrograms/ microliter). Each was dissolved in Locke-Ringer solution and each injection was 2 microliters.

The apparatus for behavioral measures was an operant chamber constructed from Formica, with inside dimensions of 48 in. long $\times 30$ in. wide $\times 36$ in. high. A bar, milk-delivery device, and photic-stimulation bulb were located in close proximity on one wall of the chamber. Preliminary training included shaping of barpressing for milk reinforcement and a shift to an FR-5 schedule, which was maintained for three 30 -min daily sessions prior to visual discrimination training. The visual discrimination task was of the "go/no-go" variety. Each trial consisted of a 10-sec reinforcement period indicated by a Grass photic stimulator flashing at $7 \mathrm{~Hz}$. During this period each barpress was reinforced with $.3 \mathrm{cc}$ of milk. Reinforcement periods were separated on a variable-interval schedule ranging from 27.5 to $33.5 \mathrm{sec}$, with a mean of $30 \mathrm{sec}$. If three responses occurred during the $10 \mathrm{sec}$ prior to the onset of the reinforcement period, the intertrial interval was extended an additional $10 \mathrm{sec}$. Ss received 50 trials per day, and records were made of total responses (TR), reinforced responses (SDR), and intertrial responses (ITR). After each daily session the ratio of intertrial responses to reinforced responses (ITR/SDR) was calculated for the 50 trials, and this ratio was utilized as an index of visual discrimination performance. Injections did not occur until an animal averaged two or less as many nonreinforced as reinforced responses (ITR/SD $R$ ratio $<$ 2.0) for 3 consecutive days. The substances were applied in a random order and there were at least $48 \mathrm{~h}$ between injection sessions. Before a baseline session qualified as a control day, the original performance criterion of ITR/SDR ratio $<2.0$ had to be met.

\section{RESULTS Histology}

At the end of the experiment each animal was sacrificed and perfused according to standard procedures. Photomicrographs were made of each placement and the accuracy of the implant was evaluated. The tips of all cannulae were within $\pm 1.0 \mathrm{~mm}$ in the anterior-posterior and lateral planes and $\pm 0.5 \mathrm{~mm}$ in the horizontal plane of the intended Horsley-Clark stereotaxic 